\title{
Purification of yersiniabactin: a siderophore and possible virulence factor of Yersinia enterocolitica
}

\author{
Hubert Haag, ${ }^{1} \dagger$ Klaus HantKe, ${ }^{2 *}$ Hartmut Drechsel, ${ }^{3}$ Igor Stojiljkovic, ${ }^{2}$ Günther Jung ${ }^{3}$ \\ and HANS ZÄHNER ${ }^{1}$ \\ ${ }^{1}$ Lehrstuhl für Mikrobiologie I, ${ }^{2}$ Lehrstuhl für Mikrobiologie II, ${ }^{3}$ Institut für Organische Chemie, Universität Tübingen, \\ Auf der Morgenstelle 28, 72076 Tübingen, Germany
}

(Received 14 January 1993; revised 6 April 1993; accepted 13 April 1993)

\begin{abstract}
HPLC analysis revealed that Yersinia enterocolitica WA-C produced two substances under iron-limiting conditions one of which was identified as 2,3-dihydroxybenzoyl-L-serine. The other compound had iron-complexing activity and was called yersiniabactin. The fur mutant $\mathrm{H1852}$ was shown to produce yersiniabactin constitutively in an iron-independent manner. Yersiniabactin was isolated by ethyl acetate extraction from the spent medium of H1852, size-fractionation chromatography and preparative HPLC. A catechol function was demonstrated with different chemical assays and by UV-visible spectroscopy. The molecular mass of yersiniabactin was determined to be $482 \mathrm{Da}$. Purified yersiniabactin stimulated growth of $Y_{+}$enterocolitica and Escherichia coli $\phi$ under ironlimiting conditions and apparently served as an iron carrier. Transport of ${ }^{55} \mathrm{Fe}$-yersiniabactin was TonB-dependent, indicating a receptor-mediated uptake across the outer membrane. A pesticin-resistant mutant missing the receptor protein FyuA was unable to transport and use yersiniabactin as a siderophore.
\end{abstract}

\section{Introduction}

Many micro-organisms excrete specific iron chelators under iron-limiting conditions. These metabolites are called siderophores, they complex iron and transport it into the cell via specific receptor proteins located in the outer membrane of Gram-negative bacteria (Braun \& Hantke, 1991). In mammalian body fluids, iron is firmly bound to carrier proteins such as lactoferrin or transferrin. This limitation of free iron in mammals is part of the unspecific immune system, the so-called nutritonal immunity (Weinberg, 1984), because the bound iron is not available for most micro-organisms attempting to colonize these environments. Pathogenic microorganisms have developed various systems to overcome this shortage of iron. In some cases the production of a siderophore is associated with increased virulence, as in the case of angiubactin produced by Vibrio anguillarum

\footnotetext{
*Author for correspondence. Tel. 07071294645 ; fax 07071294634.

$\dagger$ Present address: Sandoz Pharma AG, Building 506 Room 413, CH 4002 Basel, Switzerland.
}

Abbreviations: CAS, chromazurole S; EDDA, ethylenediamine-di(O-hydroxyphenyl acetic acid).
(Crosa, 1989). Similarly, siderophore production in Yersinia enterocolitica has been postulated to be a virulence factor (Heesemann, 1987).

Several non-pathogenic Yersiniae such as Y. frederiksenii, $Y$. intermedia and $Y$. kristensenii produce the hydroxamate-type siderophore aerobactin (Stuart $e t$ al., 1986) in contrast to the pathogenic strains of Yersiniae, which do not produce aerobactin. Iron supply in these strains is still a matter of debate. In the 1970 s conflicting results on siderophore production by the pathogenic Yersiniae were published (Perry \& Brubaker, 1979; Wake et al., 1975). Heesemann (1987) was able to demonstrate the secretion of a siderophore by virulent mouse-lethal strains of $Y$. enterocolitica (serotypes $\mathrm{O}: 8$, $\mathrm{O}: 13$ and $\mathrm{O}: 40$ ) with the colorimetric chromazurole $\mathrm{S}$ (CAS) assay (Schwyn \& Neilands, 1987). In this assay the blue CAS-iron complex is destroyed since the chelating activity of the siderophore removes the iron from the complex and the orange colour of chromazurole S appears. Strains of $Y$. pseudotuberculosis, which are mouse-lethal, were also found to be CAS-positive. However, the less virulent $Y$. enterocolitica strains of serotypes O:3, O:9, O:5.27 (Heesemann, 1987) were found to be CAS-negative. From these results it was concluded that virulent, mouse-lethal Yersiniae may produce novel siderophores that have not yet been 
detected using common siderophore assays. In addition, in common with many bacteria, $Y$, enterocolitica is able to use siderophores produced by other micro-organisms, e.g. ferrichrome, ferrioxamine $B$ and $E$, enterochelin (enterobactin) and 2,3-dihydroxybenzoyl-L-serine (Bäumler et al., 1993). The utilisation of haem via a siderophore-like uptake mechanism was demonstrated by Stojiljkovic \& Hantke (1992).

The characterization of the siderophore yersiniabactin from the culture broth of $Y$. enterocolitica $0: 8$ is described. HPLC in combination with the CAS assay was used for the detection and isolation of yersiniabactin. Furthermore, the yersiniabactin-mediated uptake of iron via the pesticin-receptor is demonstrated.

\section{Methods}

Media, growth conditions and bacteria. Yersinia enterocolitica WA-C is cured of the virulence plasmid lcr (Heesemann, 1987); WA-1 is a mutant of WA-C unable to produce yersiniabactin (Heesemann, 1987); WA-8 fyuA and WA-9 fyuA were derived from WA-1 (Bäumler $e t$ al., 1993) by selection for pesticin resistance; $\mathrm{H} 2000$ is a ton $B$ mutant derived from WA-C (Koebnik et al., 1993). For siderophore production $Y$. enterocolitica $\mathrm{H} 1852$ fur was used (Heesemann et al., 1993; Staggs \& Perry, 1992) which showed constitutive siderophore production. $Y$. enterocolitica 5030, a mutant unable to produce yersiniabactin, was selected after NTG mutagenesis and used for the bioassays. Escherichia coli $\phi$ was obtained from B. A. D. Stocker, Stanford, CA, USA.

Strains were grown on TY medium ( $8 \mathrm{~g}$ tryptone, $5 \mathrm{~g}$ yeast extract and $\left.5 \mathrm{~g} \mathrm{NaCl}^{-1}\right)$ or on nutrient broth medium $(8 \mathrm{~g}$ nutrient broth, $5 \mathrm{~g} \mathrm{NaCl}^{-1}$ ). To create iron-limiting conditions in these media ethylenediamine-di $(O$-hydroxyphenol acetic acid) (EDDA) was added at the concentrations given. The strains were stored at $-70^{\circ} \mathrm{C}$ in TY medium with $30 \%(\mathrm{v} / \mathrm{v})$ glycerol.

A modification of the medium of Cove et al. (1980) was used for siderophore production. The medium contained: A $\left(\mathrm{mg} \mathrm{1}^{-1}\right.$, final concn), L-alanine, 200; L-leucine, 200; L-arginine, 300; L-lysine, 200; L-aspartic acid, 300; L-methionine, 200; L-cystine, 50; L-phenylalanine, 200; L-cysteine, 50; L-proline, 300; L-glutamic acid, 400; L-serine, 400; L-glycine, 200; L-threonine, 300; L-histidine, 300; L-tryptophan, 200; hydroxyproline, 200; L-tyrosine, 200; L-isoleucine, 300; L-valine, 200; adenine, 20; guanine, 20; uracil, 20. $\mathrm{B}$ (mg l-1 final concn), $\mathrm{KH}_{2} \mathrm{PO}_{4}$, $1000 ;\left(\mathrm{NH}_{4}\right)_{2} \mathrm{SO}_{4}, 2000 ; \mathrm{NaCl}, 1000 ;$ MOPS, 20900, pH 7.0 adjusted with $\mathrm{NaOH}$. C, glucose $20 \mathrm{gl}^{-1}$. The solutions were combined after sterilization and $1 \mathrm{ml}$ of a sterile stock solution of vitamins $\left(\mathrm{mg} \mathrm{m}^{-1}\right.$, stock solution: folic acid, 10; biotin, 6; p-amino-benzoic acid, 200; thiamin. $\mathrm{HCl}, 1000$; panthothenic acid, 1200 ; riboflavin, 1000; nicotinic acid, 2300; pyridoxine. $\mathrm{HCl}, 12000$; cyanocobalamin, 100) and $5 \mathrm{ml}$ of a sterile solution of trace elements $\left(\mathrm{mg} \mathrm{m}^{-1}\right.$, stock solution: $\left.\mathrm{CaCl}_{2}, 1000 ; \mathrm{MgSO}_{4} \cdot 7 \mathrm{H}_{2} \mathrm{O}, 1000\right)$ were added. If necessary, traces of contaminating metals were removed by passing the medium through a Chelex 100 column prior to use. The glassware was rinsed several times with $0.1 \mathrm{M}-\mathrm{HCl}, 0.5 \mathrm{M}$-EDTA and double-distilled water. For largescale siderophore production a 4 litre stirring flask fermenter (Braun) was used.

Preparation and analysis of outer-membrane proteins by SDSPAGE, antiserum and blotting has been described by Heesemann $e t$ al. (1993).

Bioassay for siderophore detection. The bioassay was carried out by modification of the method described by Miles \& Khimji (1975). A modified production medium as described above was used. MOPS was replaced by TES $(50 \mathrm{~mm})$ and the $\mathrm{pH}$ was adjusted to $7 \cdot 5$. Deferrated EDDA (30 mg) Rogers, 1973) and $15 \mathrm{~g}$ agar (Difco) were added to solution B prior to sterilization. Molten agar $\left(10 \mathrm{ml}, 45^{\circ} \mathrm{C}\right)$ was inoculated with $30 \mu \mathrm{l}$ strain $5030\left(\mathrm{OD}_{578} 0.8\right.$ in nutrient broth) and poured into a Petri-dish. A sterile paper disc, loaded with test solution $(20 \mu \mathrm{l})$, was placed on the solidified agar and the plate was incubated at $30^{\circ} \mathrm{C}$ for $18 \mathrm{~h}$. A halo of bacterial growth surrounded those discs bearing siderophore activity or iron.

Analytical HPLC. An HP-1050 liquid chromatograph equipped with a $\mathrm{C}-18$ reverse phase column was used (Nucleosil-ODS, particle size $5 \mu \mathrm{m}, 125 \times 4.6 \mathrm{~mm}$ i.d., Grom, Herrenberg, FRG). $\mathrm{FeCl}_{3}(1 \mathrm{~mm})$ solution was added to the siderophore-containing solutions and the samples were cleared by centrifugation at $12000 \mathrm{~g}$. Samples $(20 \mu \mathrm{l})$ of these solutions were injected and separated by gradient elution. Solvent A consisted of double-distilled water with $148 \mathrm{mM}-\mathrm{H}_{3} \mathrm{PO}_{4}$, solvent $B$ was acetonitrile. The gradient was from $0-60 \%$ acetonitrile

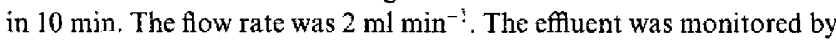
a diode-array detection system which allowed the recording of UVvisible spectra of single peaks.

Preparation of yersiniabactin. After separating the biomass by passing the culture broth through a cross-flow module (Filtron), yersiniabactin was extracted twice with an equal volume of ethyl acetate at $\mathrm{pH}$ 7. The orgunic phase containing yersiniabactin was evaporated to dryness. The residue was dissolved in methanol $(3 \mathrm{ml})$ and chromatographed on a column $(2.8 \times 40 \mathrm{~cm})$ containing Fractogel TSK HW 40 (s) with methanol as mobile phase and a flow rate of approximately $15 \mathrm{ml} \mathrm{h}^{-1}$. The siderophore-containing fractions were detected by CAS activity and HPLC. For further purification the Beckman System Gold 126 with the Waters 712 WISP (auto sampler) and a Shandon ODS Nucleosil column $(5 \mu \mathrm{m}, 300 \times 20 \mathrm{~mm})$ were used. The samples were separated by gradient elution. Solvent A was doubledistilled water, solvent $\mathrm{B}$ was acetonitrile. The gradient was $55-75 \%$ solvent $B$ in 24 min with a flow rate of $10 \mathrm{ml} \mathrm{min}{ }^{-1}$. The compound was monitored on-line by diode-array detection and checked off-line by the analytical HPLC system and the bioassay described above. The substance eluted at approx. $60 \%$ acetonitrile. Finally the yersiniabactincontaining fractions were concentrated in vacuo and lyophilized.

Spectroscopic and mass spectrometric methods. UV-visible spectra were recorded on an Ultrospec III spectrometer (Pharmacia LKB) connected to a Commodore PC40 computer. The samples were dissolved at a concentration of $0.1 \mathrm{~mm}$ in double-distilled water and the spectra were recorded in quartz cuvettes with a path length of $1 \mathrm{~cm}$ at $20^{\circ} \mathrm{C}$.

Positive FAB spectra were recorded on a Varian MAT 711 instrument coupled with an SS 200 data system. The FAB spectra were measured from a matrix of nitrobenzyl alcohol. The ion source was at $50^{\circ} \mathrm{C}$.

Electro-spray mass spectra (API-MS) were measured on a triple quadrupole mass spectrometer Sciex API III (Sciex) equipped with an Aero-Spray source operating at atmospheric pressure. The samples were dissolved in methanol with the addition of $1 \%(\mathrm{w} / \mathrm{v})$ formic acid $\left(50 \mathrm{nmol}^{-1}\right)$ and injected with a continuous flow of $2-5$ litres $\mathrm{min}^{-1}$.

Siderophore assays. The supernatant of cultures grown under ironlimiting conditions was tested by the CAS assay as described by Schwyn \& Neilands (1987). The assays for catechol or hydroxamate groups were performed as described by Arnow (1937), Csaky (1948) and Rioux et al. (1983).

Determination of glucose and ammonium. Glucose was determined on-line by HPLC as described by Plaga et al. (1989). For the determination of ammonium ions an $\mathrm{NH}_{4}^{+}$-sclective electrode was used (type M701, Orion). 
Iron transport. Cells were grown overnight at $30^{\circ} \mathrm{C}$ in nutrient broth [ $8 \mathrm{~g}$ nutrient broth (Difco), $5 \mathrm{~g} \mathrm{NaCl}^{-1}$ ] and diluted $1: 100$ into fresh nutrient broth with EDDA as indicated. After growth to a density of about $3 \times 10^{8} \mathrm{cells} \mathrm{m}^{-1}$, the cells were collected by centrifugation at $4{ }^{\circ} \mathrm{C}$, and washed once in cold M9 medium (Miller, 1972) with $0.1 \%$ (w/v) glucose and kept on ice. A suspension containing $1 \times 10^{9}$ cells (ml M9 medium ${ }^{-1}$ ) was used for the transport assay at $27^{\circ} \mathrm{C}$ in the presence of $100 \mu \mathrm{m}$-nitrilotriacetate (NTA). Desferrioxamine B was used at a concentration of $0.5 \mu \mathrm{M}$ with $0.2 \mu \mathrm{M}-{ }^{55} \mathrm{Fe}$, yersiniabactin was used at a concentration of $1 \mu \mathrm{M}$ with $0.2 \mu \mathrm{M}-{ }^{55} \mathrm{Fe}$. The 100 -fold concentrated stock solution of yersiniabactin contained $20 \%(\mathrm{v} / \mathrm{v})$ methanol, $10 \mathrm{~mm}-\mathrm{NTA}, 0.2 \mathrm{~mm}-\mathrm{NaOH}, 100 \mu \mathrm{m}$-yersiniabactin dissolved in methanol, and $20 \mu \mathrm{M}-{ }^{55} \mathrm{FeCl}_{3}, 0 \cdot 2 \mathrm{~mm}-\mathrm{HCl}$. Cells were collected on filters at the times indicated. Radioactivity was determined by liquid scintillation counting.

\section{Results}

\section{Growth under iron-restricted conditions}

$Y$. enterocolitica WA-C was grown in a defined medium treated with Chelex 100 and in an iron-enriched $(100 \mu \mathrm{M}-$ $\left.\mathrm{FeCl}_{3}\right)$ medium. Growth was decreased approximately threefold by iron-limiting conditions. The latter cultures contained CAS and biological siderophore activity. The appearance of iron-regulated metabolites was further investigated by HPLC monitoring at $320 \mathrm{~nm}$ to detect any catechol-containing siderophores and at $430 \mathrm{~nm}$ to detect any hydroxamate iron complexes. Two substances were found to be synthesized specifically under iron-limiting conditions, with retention times of 3.5 and $7.4 \mathrm{~min}$. The iron-regulated compound with a retention time of $7.4 \mathrm{~min}$ was designated yersiniabactin. By comparison with a standard reference compound, the other metabolite was found to be 2,3-dihydroxybenzoylL-serine. This compound was produced only in low amounts although after enrichment from the medium it showed growth-stimulating activity for $E$. coli and was positive in the CAS test (data not shown).

\section{Fermentation of yersiniabactin}

The influence of $\mathrm{Fe}^{3+}$ concentration on the production of yersiniabactin by $Y$. enterocolitica strains WA-C and H1852 fur are shown in Table 1. For large-scale production of yersiniabactin, the constitutive siderophore producer $Y$. enterocolitica H1852 fur (Heesemann et al., 1993) was used to avoid repression of siderophore synthesis by iron leaching from the vessel. Fig. 1 shows that the synthesis of yersiniabactin by $\mathrm{H} 1852 \mathrm{fur}$ occurred during the exponential growth phase and reached a maximum after $16 \mathrm{~h}$ with a maximum yield of $135 \mathrm{mg}^{-1}$ as anticipated for a siderophore and therefore represented type A kinetics, as described by Gaden (1959). The carbon source glucose was consumed after
$14 \mathrm{~h}$. No ammonium-suppression of yersiniabactin synthesis was observed, as has been described for antibiotic synthesis (Aharonowitz, 1980).

Table 1. Production of yersiniabactin under different conditions of iron supply

\begin{tabular}{|c|c|c|}
\hline \multirow{2}{*}{$\begin{array}{l}\mathrm{FeCl}_{3} \\
\text { added } \\
\text { to the } \\
\text { medium } \\
(\mu \mathrm{M})\end{array}$} & \multicolumn{2}{|c|}{$\begin{array}{l}\text { Yersiniabactin } \\
\qquad\left(\mathrm{mg} \mathrm{l}^{-1}\right) \\
\text { produced by: }\end{array}$} \\
\hline & $\begin{array}{l}\text { Strain } \\
\text { WA-C }\end{array}$ & $\begin{array}{l}\text { Strain } \\
\text { H1852 fur }\end{array}$ \\
\hline 0 & 101 & 112 \\
\hline 0.05 & 34 & 92 \\
\hline 1 & 36 & 94 \\
\hline 5 & 0 & 96 \\
\hline 10 & 0 & 93 \\
\hline
\end{tabular}

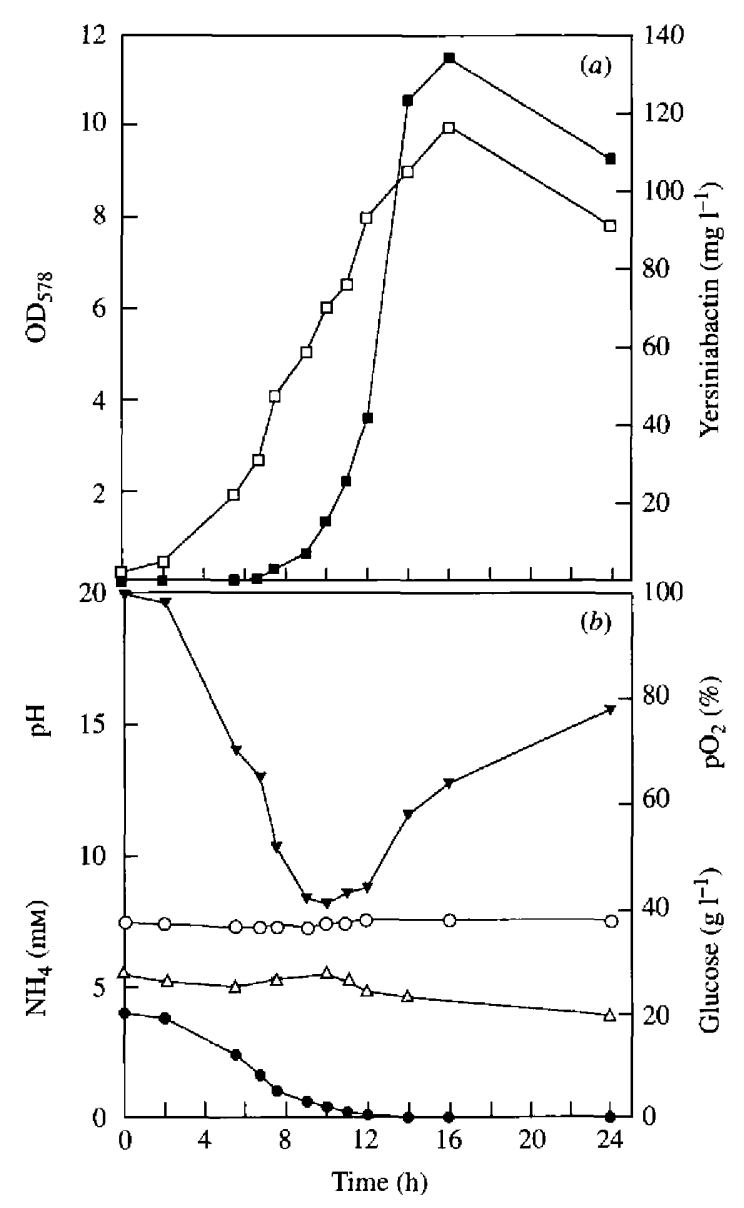

Fig. 1. (a) Production of yersiniabactin ( $\square$ ) and growth (measured as $\mathrm{OD}_{578} ; \square$ ) of strain $\mathrm{H} 1852$ fur during batch culture in modified Cove medium. Yersiniabactin was determined by analytical HPLC. (b) Utilization of substrates and the change of $\mathrm{pH}$ during fermentation. (๑) Glucose, ( $\nabla) \mathrm{pO}_{2},(\mathrm{O}) \mathrm{pH}$, and $(\triangle) \mathrm{NH}_{4}$. 


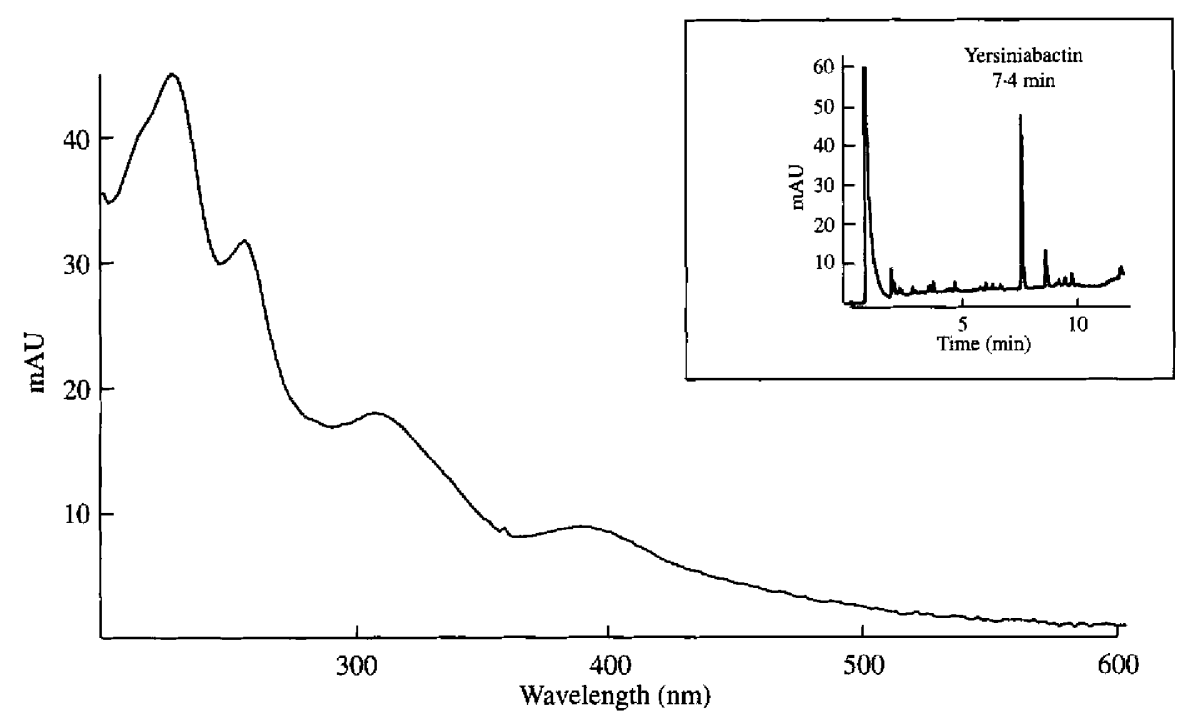

Fig. 2. UV-visible spectrum of iron-loaded yersiniabactin. The insert shows a typical HPLC run of enriched yersiniabactin.

\section{Purification of yersiniabactin}

The iron-chelating metabolite was purified in the desferri form. Preliminary studies with non-ionic polymeric adsorbents indicated adsorption to XAD 2 and XAD 4. However, elution with methanol containing $1 \%(\mathrm{w} / \mathrm{v})$ formate led to several degradation products. For this reason yersiniabactin was extracted from the neutralized culture supernatant with ethyl acetate. The organic phase was evaporated to dryness and redissolved in methanol for size-fractionation on Fraktogel. Siderophore-containing fractions were detected by HPLC with monitoring at $210 \mathrm{~nm}$ and the CAS assay. Pure material was obtained using preparative HPLC with a water/acetonitrile gradient: yersiniabactin eluted at about $60 \%$ acetonitrile. The siderophore-containing fractions were concentrated by evaporation and lyophilized to dryness. A colourless powder (approx. 40\% yield) was obtained.

\section{Physico-chemical characterization of yersiniabactin}

Atmospheric pressure ion-spray mass spectra showed that yersiniabactin has a molecular mass of $482 \mathrm{Da}$ and could easily be divided into two main fragments of $295 \mathrm{Da}$ and $190 \mathrm{Da}$. The purified compound showed CAS- and growth-stimulating activity for $Y$. enterocolitica 5030 on EDDA plates. Using Csaky's (1948) method, no hydroxamate residues were detected in the siderophore. According to the tests of Arnow (1937) and Rioux et al. (1983), the presence of catechol groups could be demonstrated without ambiguity. However, the yersiniabactin-iron complex was colourless, in contrast to the wine-red iron complex of enterochelin (enterobactin), indicating the presence of additional non- catechol-containing iron-chelating groups. For UV spectroscopy the isolated compound was dissolved at a concentration of $1 \mathrm{~mm}$ (molecular mass $482 \mathrm{Da}$ ) in double-distilled water. The spectra, without iron and as a $1: 1$ complex with iron, were recorded at different $\mathrm{pH}$ values. The desferri ligand showed absorption maxima at 210,260 and $320 \mathrm{~nm}$. The addition of iron resulted in a charge-transfer band at $400 \mathrm{~nm}$ which had already been observed in the diode-array spectrum (Fig. 2). This supported the assumption that it is not a pure catecholate-type complex which would absorb at about $500 \mathrm{~nm}$. Variation of the $\mathrm{pH}$ between 3.7 and 8.9 did not significantly alter the spectrum.

\section{Iron transport with yersiniabactin}

$Y$. enterocolitica WA-C was grown to about $3 \times 10^{8}$ cells $\mathrm{ml}^{-1}$ in nutrient broth to which $20 \mu \mathrm{M}$-EDDA had been added to derepress the iron transport systems. Iron(III) uptake was observed with yersiniabactin and ferrioxamine B (Fig. 3). Without addition of EDDA, very low uptake rates were observed indicating that an excess of iron in the medium led to repression of both transport systems. As a control, iron transport was measured in the TonB mutant $Y$. enterocolitica $\mathrm{H} 2000$ (Koebnik et al., 1993) which was grown in the same medium with only $10 \mu \mathrm{M}$-EDDA added to allow sufficient growth of the cells. No uptake was observed with ferrioxamine B as would be expected for a TonB mutant. Also no uptake was observed with yersiniabactin, indicating TonB and receptor-dependent uptake.

The pesticin-resistant strains WA-8 and WA-9 lacked the outer-membrane protein FyuA (Fig. 4a). These strains were derived from WA-1 which showed a very low expression of FyuA (Fig. 4a). This was confirmed by 


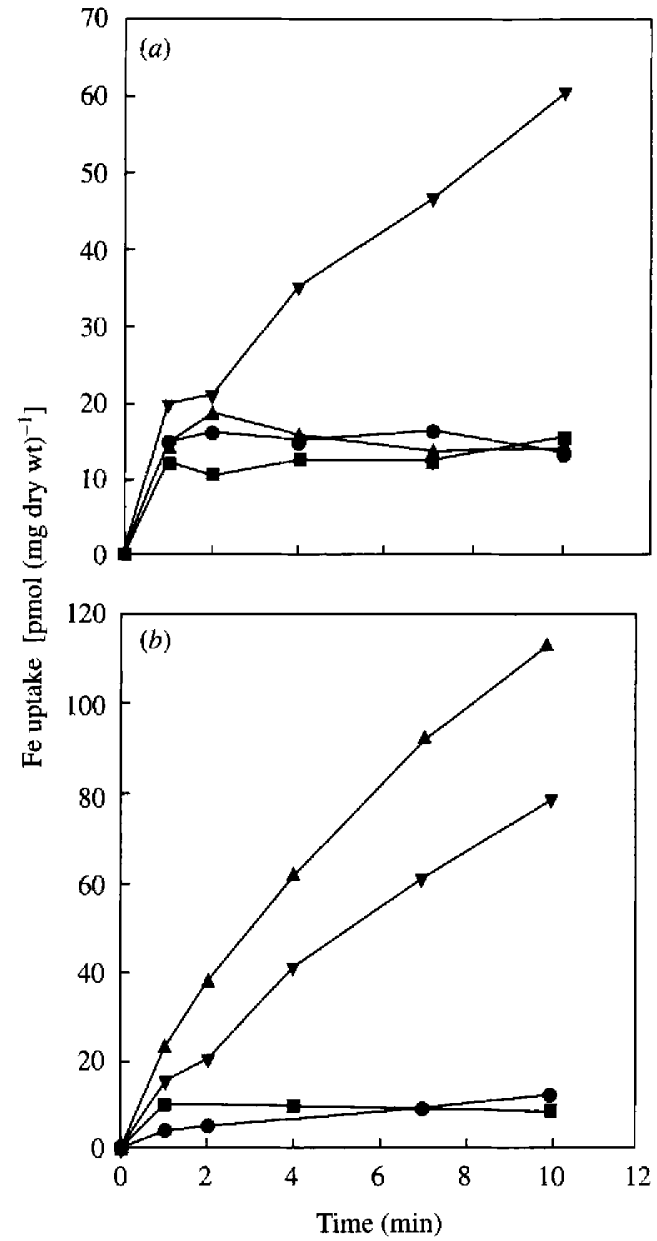

Fig. 3. Iron uptake with yersiniabactin $(a)$ and ferrioxamine $\mathrm{B}(b)$ as a siderophore. (-) Strain WA-C, ( $)$ strain WA-C grown with $20 \mu \mathrm{M}-$ EDDA, (A) WA-8 fyuA grown with $10 \mu \mathrm{M}-\mathrm{EDDA}$, and (ם) H2000 ton $B$ grown with $10 \mu \mathrm{M}$-EDDA. A representative example of three experiments is shown.

blotting and probing with specific antibodies against FyuA (Fig. $4 b$ ). The antibodies reacted with the FyuA band from WA-C and a smaller band was visible with the membrane from WA-1. No cross-reacting material was visible with strains WA-8 and WA-9. Good ferrioxamine B mediated iron uptake was observed with strain WA-8. However, no yersiniabactin iron was taken up (Fig. 3), indicating that the pesticin receptor FyuA is also the receptor for yersiniabactin.

There are only two strains of $E$. coli described in the literature which are pesticin sensitive, E. coli $\phi$ and CA42 (Ferber et al., 1981). E. coli $\phi$ was able to grow in the bioassay for siderophore detection with yersiniabactin in contrast to $E$. coli $\mathrm{K} 12$. In addition, $E$. coli $\phi$ was able to transport ${ }^{55} \mathrm{Fe}$-yersiniabactin with similar efficiency to $Y$. enterocolitica WA-C (data not shown). This gives additional evidence that the pesticin receptor is the receptor for yersiniabactin.

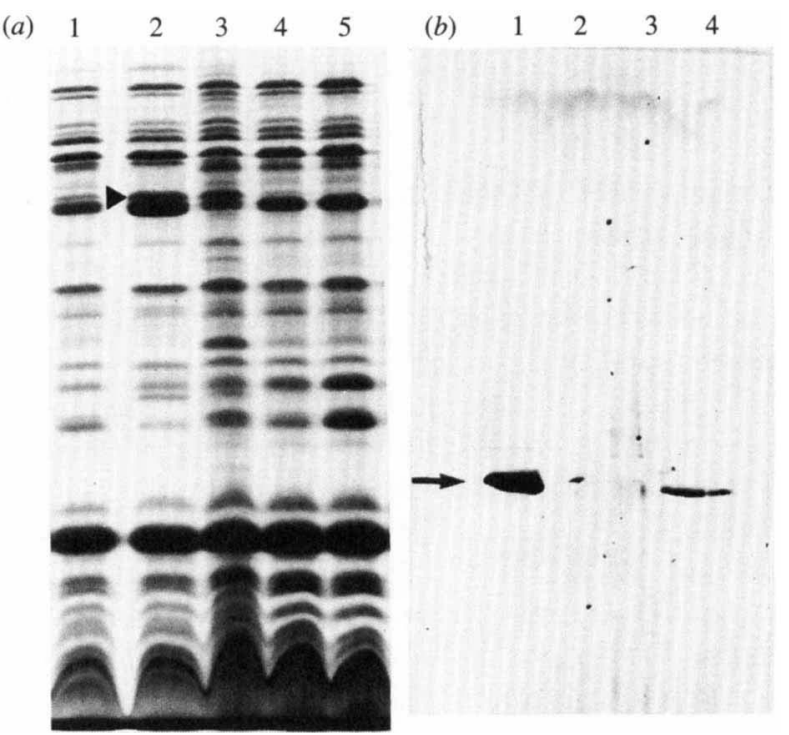

Fig. 4. (a) Outer membrane proteins from different Yersinia strains separated by SDS-PAGE. Lanes: 1, WA-C grown on TY medium (iron rich); 2, WA-C grown on nutrient broth with $20 \mu \mathrm{M}$-EDDA; 3-5, strains H1852 fur (3), WA1 (4) and WA-8 (5) grown on nutrient broth with $10 \mu \mathrm{M}$-EDDA. The position of FyuA is indicated by a triangle. (b) Immunoblot of outer-membrane proteins separated by SDS-PAGE and developed with anti-FyuA serum. The proteins were from (1) Yersinia enterocolitica WA-C, (2) WA-8, (3) WA-9 and (4) WA-1. Preparation of outer membranes, SDS-PAGE, blotting and the antiserum have been described by Heesemann et al. (1993).

\section{Discussion}

It is now more than 15 years since Wake et al. (1975) published the first observation that $Y$.pestis may produce a siderophore. Under iron-limiting conditions 'iron responsive' material was isolated from $Y$. pestis by Perry \& Brubaker (1979). This compound had no biological activity which led the authors to conclude that there is no siderophore produced by $Y$. pestis. It was only recently that the siderophore activity was rediscovered in a group of highly pathogenic Yersiniae (Heesemann, 1987).

A combination of three different assays, bioassay, colorimetric CAS assay and the screening for ironregulated peaks by HPLC proved to be a powerful tool in the detection of iron complexing metabolites. Two compounds were found in the supernatant of $Y$. enterocolitica, 2,3-dihydroxybenzoyl-L-serine as a minor component and yersiniabactin as the main product. In fermenters where it was difficult to establish iron-limiting growth conditions, the fur mutant of $Y$. enterocolitica proved to be a good choice for the isolation of yersiniabactin from large-scale cultures.

Isolation of yersiniabactin by adsorption to the nonionic adsorbents of the XAD type was inappropriate since the siderophore was destroyed during desorption from this material. Similar problems may have occurred during the work of Perry \& Brubaker (1979) when they 
tried to elute the substance from charcoal. Extraction with ethyl acetate followed by HPLC in a water/ acetonitrile gradient proved to be a simple and efficient isolation procedure.

Yersiniabactin caused a halo of growth in the biological assays with $Y$. enterocolitica $\mathrm{H} 5030$ and $E$. coli $\phi$ as indicator strains. The preliminary chemical characterization of yersiniabactin showed that it is a relatively small siderophore with a molecular mass of $482 \mathrm{Da}$ which contains at least one catechol moiety. Further evidence for a catechol-containing siderophore was the observation that the aro $A$ mutant $Y$. enterocolitica YAM-1 was unable to produce yersiniabactin (Heesemann et al., 1993). The other coordination sites for iron(III) are still to be elucidated. Yersiniabactin mediated the uptake of ${ }^{55} \mathrm{Fe}$ into $Y$. enterocolitica $\mathrm{O}: 8$ strain WA-C in about the same amount as ferrioxamine B. Relatively high binding of ${ }^{55} \mathrm{Fe}$ to the cells was observed in the transport assay in contrast to the uptake experiments with ferrioxamine B. One possible reason is the hydrophobic nature of yersiniabactin which may enhance non-specific binding to the cell surface.

The transport experiments showed that the uptake of yersiniabactin -iron was regulated by the iron supply of the cell. In addition, transport was TonB-dependent as is usually the case for siderophore-mediated iron uptake in enteric bacteria (Braun \& Hantke, 1991).

It has been claimed that the pesticin receptor FyuA found in $Y$.pestis, Y.pseudotuberculosis and in certain $Y$. enterocolitica strains is the receptor for yersiniabactin (Heesemann et al., 1993). In the present work we have shown that a mutant lacking the pesticin receptor FyuA was unable to use yersiniabactin as a siderophore. Additional evidence was obtained from experiments with $E$. coli $\phi$, one of the rare $E$. coli strains sensitive to pesticin. This strain was able to use and transport yersiniabactin as a siderophore in contrast to $E$. coli $\mathrm{K} 12$ which was unable to grow with yersiniabactin as an iron source. The pesticin receptor FyuA therefore appears to be responsible for transport of the yersiniabactin-iron complex.

Members of $Y$. enterocolitica can be divided into two groups of strains with respect to their pathogenicity: the so-called mouse-lethal serotypes $(\mathrm{O}: 8, \mathrm{O}: 13, \mathrm{O}: 40)$ and the less pathogenic ones $(0: 9,0: 5)$. Robins-Browne \& Prpic (1985) showed that mouse lethality can be conferred on the less pathogenic strains by supplementation with ferrioxamine $B$. This suggests that the efficiency of iron acquisition determines the degree of pathogenicity. A major difference between these two groups of strains is their sensitivity to pesticin and ability to produce yersiniabactin (Heesemann et al., 1993). The less pathogenic strains are pesticin-resistant and do not produce yersiniabactin. It is therefore probable that yersiniabactin is an important virulence factor for $Y$. enterocolitica.

Pesticin sensitivity is also found in $Y$. pestis and in $Y$. pseudotuberculosis (Ferber et al., 1981; Heesemann et al., 1993). For these strains there is evidence that they also produce a siderophore (Heesemann, 1987; Heesemann et al., 1993; Wake et al., 1975; Perry \& Brubaker, 1979). Since the pesticin receptor is the receptor for yersiniabactin, it is highly likely that they all produce the siderophore yersiniabactin described in the present paper.

This work was supported by the SFB 323. We thank Dr Taraz and Professor Dr Budzikiewicz, University of Cologne, Germany, for measuring high-resolution FAB-MS. We also thank J. Heesemann (Institut für Hygiene, Würzburg) for antiserum and V. Braun and A. Griffin for critical reading of the manuscript.

\section{References}

Aharonowitz, Y. (1980). Nitrogen metabolite regulation of antibiotic biosynthesis. Annual Review of Microbiology 34, 209-233.

ARNow, L. E. (1937). Colorimetric determination of the components of 3,4-dihydroxy-phenylalanine-tyrosine mixtures. Journal of Biological Chemistry 118, 531-537.

Bäumler, A., Koebnik, R., Stojlljkovic, 1., Heesemann, J., Braun, V. \& HANTKE, K. (1993). Survey on newly characterized iron uptake systems of Yersinia enterocolitica. Zentralblatt für Mikrobiologie 278, 416-424.

BRAUN, V. \& HANTKE, K. (1991). Genetics of bacterial iron transport. In Handbook of Bacterial Iron Chelates, pp. 107-183. Edited by G. Winkelmann. Boca Raton: CRC Press.

Cove, J. H., Holland, K. T. \& CunlifF, W. J. (1980). The vitamin requirements of staphylococci isolated from the human skin. Journal of Applied Bacteriology 49, 29-37.

Crosa, J. H. (1989). Genetics and molecular biology of siderophoremediated iron transport in bacteria. Microbiological Reviews 53, $517-530$.

CZaKy, T.Z. (1948). On the estimation of bound hydroxylamine in biological materials. Acta Chemica Scandinavica 2, 450-454.

Ferber, D. M., Fowler, J. M. \& BrubackeR, R. R. (1981). Mutations to tolerance and resistance to pesticin and colicins in Escherichia coli $\phi$. Journal of Bacteriology 146, 506-511.

GADEN, E. L. (1959). Fermentation process kinetics. Journal of Biochemical and Microbiological Techniques and Engineering 1, 413-442.

HeestimanN, J. (1987). Chromosomal encoded siderophores are required for mouse virulence of enteropathogenic Yersinia species. FEMS Microbiology Letters 48, 229-233.

Heesemann, J., Vocke, T., Hantke, K., Saken, E., Stonllukovic, I., RAKIN, A. \& BERNER, R. (1993). Virulence of Yersinia enterocolitica is closely associated with siderophore production, expression of an iron repressible outer membrane polypeptide of 65000 daltons and pesticin-sensitivity. Molecular Microbiology 8, 397-408.

Koebnik, R., Bäumler, A., Hebsemann, J., Braun, V. \& Hantke, K. (1993). The TonB protein of Yersinia enterocolitica and its interactions with TonB-box proteins. Molecular and General Genetics 237, $152-160$.

Miles, A. A. \& KHIMIr, P. L. (1975). Enterobacteriaj chelators of iron: their occurrence, detection, and relation to pathogenicity. Journal of Medical Microbiology 8, 477-490.

MiLler, J. H. (1972). Experiments in Molecular Genetics. Cold Spring Harbor, NY: Cold Spring Harbor Laboratory.

PerRy, R. D. \& BRUbaker, R. R. (1979). Accumulation of iron by Yersiniae. Journal of Bacteriology 137, 1290-1298.

Plaga, A., Stümpfel, J. \& FiedLer, H. P. (1989). Determination of 
carbohydrates in fermentation processes by high performance liquid chromatography. Applied Microbiology and Biotechnology 32, 45-49. Rioux, C., Jordan, D. C. \& RatTray, J. B. M. (1983). Colorimetric determination of catechol siderophores in microbial cultures. Analytical Biochemistry 133, 163-169.

Robins-Browne, R. M. \& PrPIC, J. K. (1985). Effects of iron and desferrioxamine on infections with Yersinia enterocolitica. Infection and Immunity 47, 779-794.

Rogers, H. J. (1973). Iron-binding catechols and virulence in Escherichia coli. Infection and Immunity 7, 445-456.

SCHWYN, B. \& NEILANDS, J. B. (1987). Universal chemical assay for the detection and determination of siderophores. Analytical Biochemistry 160, $47-56$
Staggs, T. M. \& Perre, R. D. (1992). Fur regulation in Yersinia species. Molecular Microbiology 6, 2507-2516.

STOJILJKovic, I. \& HANTKE, K. (1992). Hemin uptake of Yersinia enterocolitica: similarities with other Ton B dependent systems in gram-negative bacteria. EMBO Journal 11, 4359-4367.

Stuart, S., Prpic, J. K. \& Robins-Browne, R. M. (1986). Production of aerobactin by some species of the genus Yersinia. Journal of Bacteriology 166, 1131-1133.

Wake, A., Misawara, M. \& Matsui, A. (1975). Siderochrome production in Yersinia pestis and its relation to virulence. Infection and Immunity 12, 1211-1213.

WEINBERG, E. D. (1984). Iron and infection. Microbiological Reviews 42, 4566 . 